\title{
A Low-Complexity Multiuser Detector for Asynchronous CDMA QPSK Adaptive Antenna Array Systems
}

\author{
E. DEL RE, R. FANTACCI, S. MOROSI* and S. MARAPODI \\ Department of Electronics and Telecommunications, University of Florence, 50139 Florence, Italy
}

\begin{abstract}
This paper proposes a low complexity joint space-time multiuser detection algorithm for asynchronous DS/CDMA antenna array systems. The proposed multiuser detector is composed of an adaptive antenna array, used as a linear beamformer, and a sliding window decorrelator. A QPSK modulation scheme is used in order to increase bandwidth efficiency. Numerical results are given in terms of Bit Error Rate (BER) under the assumption of a frequency-selective Rayleigh slow fading channel. In particular, the proposed receiver is shown to be near-far resistant, even in worst fading cases, and to exploit completely array introduction while maintaining acceptable computational complexity. The proposed architecture avoids linear filter realization of the decorrelator, which is impractical in the case of a large number of users, and operates with relatively short data frames instead of the complete information sequence. Finally, this receiver is very flexible to changes in timing configuration.
\end{abstract}

Keywords: Direct Sequence Code Division Multiple Access (DS-CDMA), adaptive antenna systems, Multiuser Detection (MUD), decorrelating detector

\section{Introduction}

Recently, wireless communication standardization process has put thrust in using Direct Sequence Code Division Multiple Access (DS-CDMA) techniques for 3rd generation of wireless systems: main targets of these systems will be the integration of different types of traffic at a variable bit-rate with optimum resources management. DS-CDMA appears to be attractive for such applications because its characteristics include large potential capacity increases and other technical factors such as anti-multipath fading capabilities. Major hurdles of DS-CDMA systems are the near-far problem and the multiple-access interference (MAI) that limit the number of active users in relation to a specified BER [1]. Commercial digital cellular systems based on CDMA, like IS-95A by EIA/TIA [10], use stringent power control to combat near-far effects and fading [5].

An alternative solution can be identified in the multiuser detection [11] that treats co-channel interference as an additional source of information. This technique mitigates the near-far effect and, at the same time, significantly raises capacity by facing multiple access interference impairments. Lupas and Verdù [8] proposed a near-far resistant multiuser receiver, generally known as decorrelating detector, derived by considering the received signal as the sum of all the asynchronous transmitted signals and achieving perfect demodulation for each active user in an AWGN environment. Although decorrelating algorithms offer huge potential capacity and significantly improved performance, alleviating the disadvantages associated with the conventional scheme, its complexity is not sustainable for practical systems. This fact has motivated the research of suboptimal low-complexity approaches like the Sliding Window Algorithm (SWA) $[7,13]$.

\footnotetext{
* Corresponding author.

E-mail: morosi@lenst.det.unifi.it
}

Wireless cellular communication systems are characterized by random location of the users around the base station: this makes possible to employ the information about direction of arrival of the signals to improve the multiuser detector performance through adaptive antenna array introduction. Antenna array systems have been generally used so far to improve signals from a particular user or to reduce interference from other directions, independently of the demodulation technique.

Multiuser detection techniques, coupled with spatial processing through the use of an antenna array $[9,12]$ make maximum exploitation of the information at the receiver input possible. Moreover, Spread Spectrum techniques allow the receiver to discriminate each signal replica, considered here as independent virtual users, and, after space and time processing, to combine every contribution of the same signal, as in a classical rake receiver.

In this paper, we propose a near-far resistant space time multiuser detector of QPSK signals. The receiver is based on a generalized implementation of the SWA for the adaptive antenna array architecture: this algorithm is based on the periodic insertion of a zero-energy bit in the information sequence in order to perform decorrelation only for a short subsequence; window length has been chosen so that implementation complexity remains moderate. This low-complexity multiuser approach enables the separation of multiple cochannel user signals impinging on a base station antenna array. User signals are separated both in space, i.e., according to different Angle of Arrival (AOA) values, and in time, i.e., in dependence of different random delays and Time Of Arrival (TOA) values.

Our simulations are based on a suburban type propagation channel as described in GSM Recommendations [6]: the frequency-selective slow-fading channel is modeled with six 
distinct paths: the phase and amplitude of each path vary with a uniform and Rayleigh distribution, respectively. In this slow fading environment, channel parameters may be assumed constant for some bits, so that it is possible to efficiently implement SWA if the processing window length is not too long [2].

The paper is organized as follows. Section 2 describes the system and channel models together with the proposed receiver scheme; section 3 compares the BER performance of our low-complexity approach with that of a classical rake receiver employing the same antenna array configuration. Finally, section 4 concludes the paper.

\section{System model}

In the following, we focus on a communication system with $K$ simultaneous asynchronous users, each transmitting a data packet formed by $N$ bits. As in W-CDMA standard for 3G mobile communications [4], QPSK modulated signals are considered with higher bandwidth efficiency in comparison with classical binary signaling.

We assume that $L$ multipath components of each user are resolved at the receiving end. The phase, time delay and direction of arrival of each path are assumed to be perfectly known whereas no hypothesis is made about the path attenuation. Without loss of generality each replica of each user is considered as a virtual single replica user so that the total number of virtual users to be processed becomes $K L$.

The antenna array at the receiver is formed by $P$ identical omnidirectional elements. Direction vector of the $i$ th virtual user is denoted by

$$
\mathbf{v}_{i}=\left[\mathrm{e}^{-\mathrm{j} \phi_{i_{1}}}, \mathrm{e}^{-\mathrm{j} \phi_{i_{2}}}, \ldots, \mathrm{e}^{-\mathrm{j} \phi_{i} P}\right]^{T} \quad \text { where } i=1, \ldots, K L .
$$

Hence, the relative phase of the $p$ th element with respect to the AOA of the equivalent $i$ th virtual user is given by $\phi_{i_{p}}$ : in particular, the antenna system is composed of three linear uniform arrays, each covering a sector of $120^{\circ}$, with one, two and four elements per array. However, the multiuser detection approach presented in this paper is general and can be easily extended to the case of a particular array of elements, even with directional properties, with all virtual users received from a single sector within the main lobe [9]. Note that, if elements are modeled as omnidirectional elements, it is easier to derive essential array properties, even incorporating element directivity factors.

Let us introduce cross-correlation fundamental matrices $R(j), \quad j=-1,0,1$ [8], whose elements $R_{i, m}(j), i=$ $1, \ldots, K L, m=1, \ldots, K L$, are:

$$
R_{i, m}(j)=\int_{-\infty}^{\infty} \tilde{s}_{i}\left(t-\tau_{i}\right) \tilde{s}_{m}\left(t-\tau_{m}-j T\right) \mathrm{d} t
$$

where $\tilde{s}_{i}$ and $\tau_{i}$ are the normalized signature waveform and the delay of the $i$ th virtual user, respectively, and $T$ is the symbol time.

In the single element case, assuming the same spreading sequence for the in-phase and quadrature components of the transmitted QPSK signal, the matched filter output for the $n$th bit of all users is written as [8]:

$$
\begin{aligned}
\mathbf{y}(n)= & \mathbf{R}(-1) \mathbf{C}(n+1) \mathbf{b}(n+1)+\mathbf{R}(0) \mathbf{C}(n) \mathbf{b}(n) \\
& +\mathbf{R}(1) \mathbf{C}(n-1) \mathbf{b}(n-1)+\mathbf{n}(n)
\end{aligned}
$$

where the complex vector $\mathbf{b}(n)=\mathbf{b}_{I}(n)+\mathrm{j} \mathbf{b}_{Q}(n)$ is composed of the in-phase and quadrature transmitted data,

$$
\begin{gathered}
\mathbf{b}_{I}(n)=\left[\begin{array}{lll}
b_{I, 1}(n) & \ldots & b_{I, K L}(n)
\end{array}\right]^{T}, \\
\mathbf{b}_{Q}(n)=\left[\begin{array}{lll}
b_{Q, 1}(n) & \ldots & b_{Q, K L}(n)
\end{array}\right]^{T}, \\
b_{I, i}(n), b_{Q, i}(n) \in\{ \pm 1\},
\end{gathered}
$$

the complex vector $\mathbf{y}(n)=\mathbf{y}_{I}(n)+\mathrm{j} \mathbf{y}_{Q}(n)$ is composed of the in-phase and quadrature matched filter outputs, $\mathbf{C}(n)$ are the diagonal matrices whose elements, $c_{i}(n)$, are the complex channel fading gains for $i=1, \ldots, K L, n=1, \ldots, N$, with $N$ equal to the complete received sequence length. As shown in [8], the complex vector $\mathbf{n}(n)$ is the additive Gaussian noise vector, with a zero mean and covariance matrix given by

$$
E\left[\mathbf{n}^{H}(i) \mathbf{n}(j)\right]=\sigma^{2} \mathbf{R}(i-j)
$$

where superscript ${ }^{H}$ denotes complex conjugate transpose. Note that bit and chip shapes are assumed to be rectangular; moreover $c_{i}(n)=\sqrt{w_{i}(n)} \cdot \mathrm{e}^{\mathrm{j} \theta_{i}(n)}$, where $w_{i}(n)$ is the received power and the phase term $\theta_{i}(n)$ takes into account phase fluctuations due to fading. While perfect knowledge of $\theta_{i}(n)$ is assumed, $w_{i}(n)$ has an unknown value.

Let us consider a multi-element antenna with linear beamformer $\mathbf{T}_{A}: C^{P} \rightarrow C^{K L}$ in order to map the received $P$ signals into $K L$ linear combinations and to define spatial correlation coefficients $\xi_{i m}=\mathbf{v}_{i}^{H} \mathbf{v}_{m}$. Let $\mathbf{A}$ be the $P \times K L$ direction matrix given by $\left(\mathbf{v}_{1}, \ldots, \mathbf{v}_{K L}\right)$ and $\mathbf{T}_{A}$ be equal to $\mathbf{A}^{H}$.

It has been demonstrated [9] that, in multielement systems, cross-correlation fundamental matrices (1) undergo the following linear transformation:

$$
\mathbf{M}(j)=\mathbf{R}(j) \circ\left(\mathbf{A}^{H} \mathbf{A}\right)
$$

where operator $\circ$ denotes the element by element product or Hadamard product. ${ }^{1}$ Hence, for a receiver with multielement antenna and linear beamformer, matched filter outputs (2) can be expressed as:

$$
\begin{aligned}
\mathbf{y}(n)= & \mathbf{M}(-1) \mathbf{C}(n+1) \mathbf{b}(n+1)+\mathbf{M}(0) \mathbf{C}(n) \mathbf{b}(n) \\
& +\mathbf{M}(1) \mathbf{C}(n-1) \mathbf{b}(n-1)+\mathbf{n}(n) .
\end{aligned}
$$

Therefore, it is possible to eliminate the mutual interference for each virtual user by resorting to a decorrelating detector [8]. As it has been stated before, each individual replica is considered as a virtual user, so that it is possible to take advantage of both spatial processing and rake combining. Wideband linear beamforming is introduced in order to achieve coherent linear combining of each element output for each replica of each user to the joint optimum multiuser detection purpose. The architecture of the proposed receiver is shown in

\footnotetext{
${ }^{1}$ Every element of $\mathbf{M}(j)$ can be expressed as $M_{i m}(j)=R_{i m}(j)$ $\sum_{h=1}^{P}\left(A_{h i}\right)^{*} A_{h m}=R_{i m}(j) \cdot \xi_{i m}$
} 


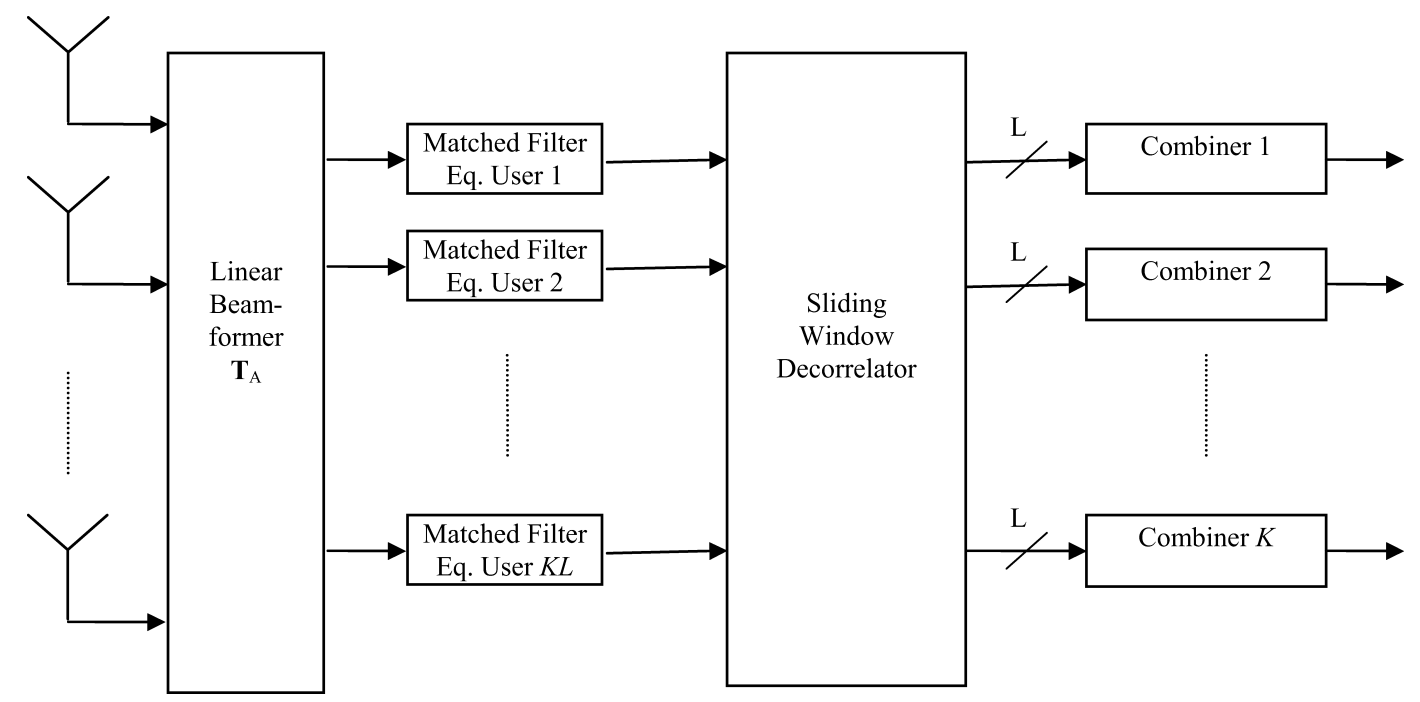

Figure 1. Receiver block diagram.

figure 1. It is important to point out that computational complexity of proposed receiver is nearly constant if an higher number of array elements is considered: in fact, from (3) it can be deduced that crosscorrelation matrix $\mathbf{M}(j)$ dimension is still equal to $K L$ and can be determined via simple matrix products.

Even though the complexity of this receiver is lower than that of an optimum receiver, it is still not low enough too allow an effective implementation. In particular, an $N K L \times N K L$ complex matrix inversion is necessary. This operation in practical applications results to be too computationally expensive. As an alternative, a Linear Time-Invariant (LTI) filter implementation of the decorrelator can be introduced [8] but also this solution is not viable, even for a modest number of users.

In this paper we propose a low-complexity implementation of a multielement decorrelator antenna with regard to parameter $N$. In our SWA, instead of the entire informative sequence, only a subsequence formed by $\widehat{N}$ bits, i.e., a "window", is considered. For simplicity we define

$$
\tilde{\mathbf{b}}(n)=\mathbf{C}(n) \cdot \mathbf{b}(n) .
$$

By applying (4) to all the $\widehat{N}$ bits of the window, i.e., for $n=$ $U+1, \ldots, U+\widehat{N}$, we have:

$$
\left[\begin{array}{c}
\mathbf{y}(U+1) \\
\mathbf{y}(U+2) \\
\mathbf{y}(U+3) \\
\vdots \\
\mathbf{y}(U+\widehat{N})
\end{array}\right]=
$$$$
\left[\begin{array}{ccccc}
\mathbf{M}(0) & \mathbf{M}(-1) & 0 & \ldots & 0 \\
\mathbf{M}(1) & \mathbf{M}(0) & \mathbf{M}(-1) & \ldots & 0 \\
0 & \mathbf{M}(1) & \mathbf{M}(0) & \ldots & 0 \\
\vdots & \vdots & \ddots & \ddots & \vdots \\
0 & \ldots & \ldots & \mathbf{M}(1) & \mathbf{M}(0)
\end{array}\right]\left[\begin{array}{c}
\tilde{\mathbf{b}}(U+1) \\
\tilde{\mathbf{b}}(U+2) \\
\tilde{\mathbf{b}}(U+3) \\
\vdots \\
\tilde{\mathbf{b}}(U+\widehat{N})
\end{array}\right]
$$

$$
+\left[\begin{array}{c}
\mathbf{M}(1) \tilde{\mathbf{b}}(U) \\
0 \\
\vdots \\
0 \\
\mathbf{M}(-1) \tilde{\mathbf{b}}(U+\widehat{N}+1)
\end{array}\right]+\left[\begin{array}{c}
\mathbf{n}(U+1) \\
\mathbf{n}(U+2) \\
\mathbf{n}(U+3) \\
\vdots \\
\mathbf{n}(U+\widehat{N})
\end{array}\right]
$$

where $U$ is an offset from the start of the transmission indicating the beginning of the considered window and $\widehat{N}<N$ is the data window temporal length within the complete received sequence. The linear system (6) is composed of $\widehat{N}$ equations with $\widehat{N}+2$ unknown values: in particular, $\mathbf{y}(U+1)$ is dependent on $\tilde{\mathbf{b}}(U)$ and, likewise, $\mathbf{y}(U+\widehat{N})$ on $\tilde{\mathbf{b}}(U+\widehat{N}+1)$.

Our approach is to assume terms $\tilde{\mathbf{b}}(U)$ and $\tilde{\mathbf{b}}(U+\widehat{N}+1)$ in (6) equal to zero (figure 2). This condition is fulfilled by periodically inserting a zero-energy bit in the information bit sequence. Therefore, the interference due to the crosscorrelation of the actual symbols with the past and future symbols in the asynchronous channels can be eliminated. Parameter $\widehat{N}$ has to be chosen in order to obtain a feasible (lowcomplexity) solution of (6). Moreover, the transmission is arranged so that the relative differences in arrival times of the zero-energy bits at the base-station are smaller than the single-bit duration $T_{\mathrm{b}}$,

$$
\left|\tau_{i}-\tau_{m}\right| \leq T_{\mathrm{b}}, \quad \forall i, m .
$$

It is important to note that the overhead needed to fulfill the synchronization constraints is not too high [3] and that this loose constraint on relative delays give the receiver great flexibility towards change in timing configuration. Now, by exploiting the block tridiagonal structure of $\mathbf{M}$ according to the algorithm outlined in [3], we can solve the linear system (6).

Performing matrix inversion on the short window frame simplifies computational and hardware requirements in comparison to approaches based on a linear filter implementation. 


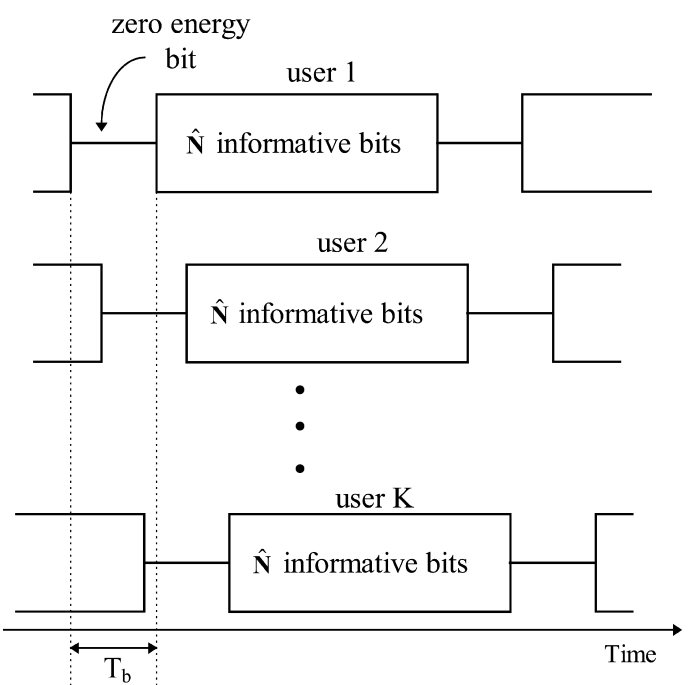

Figure 2. Transmitted data frame structure.

If the SWA is utilized, computational $\operatorname{load}^{2}$ needed for the inversion of the matrix $\mathbf{M}$ is on the order of $O\left((K L)^{2}\right)$, avoiding the realization of $(K L)^{2}$ IIR filters required by a classical decorrelator [14]. Moreover, the adaptive array introduction does not cause an increase in the dimensions of the matrix $\mathbf{M}$, so that its complexity remains equal to the case of the singleelement antenna. In this case, only a negligible increase of the computational load is caused by linear beamformer introduction.

The decorrelation block outputs $\tilde{\mathbf{b}}(n)$ are mutually interference free but are dependent on the random phase offset introduced by the multipath fading channel. In order to implement a coherent QPSK detection, we need to compensate this random phase offset. Since each operation on the received signal is linear, it is possible to easily accomplish this task after the decorrelation operation.

Finally, by combining all the replicas, the values of the decision variables are obtained. We emphasize that only AWGN and attenuation due to multipath fading propagation affect them while MAI and random phase fluctuations are effectively faced by the proposed multiuser detector.

\section{Simulation results}

In this section, numerical results concerning the BER performance of the proposed receiver are presented and compared with those obtained by a classical rake detector in the case of different interference environments. We made the following assumptions in our simulations:

- QPSK modulated data with symbol rate equal to 31.496 ksymbols/s;

- Individual spreading sequence assigned to each QPSK user;

2 If matrix $\mathbf{M}$ does not need to be updated, i.e., if fading fluctuations are not too fast, computational load will be reduced to $O(K L)$.

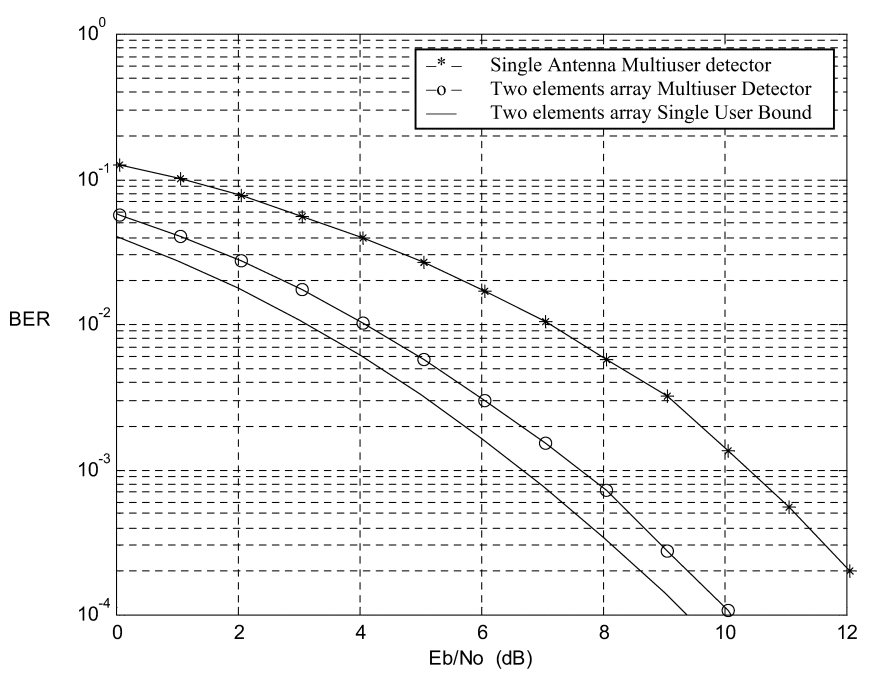

Figure 3. Multiuser detector BER comparisons (ideal power control, antennas with one and two elements).

- Spreading obtained through Gold sequences with processing gain equal to 127 .

We have considered 4 active users in up-link communications and a Rayleigh multipath fading channel, as specified in [6], with six resolvable paths and a Doppler spread of $100 \mathrm{~Hz}$. By taking into account that BER performance of the proposed receiver is independent of $\widehat{N}$ [2], we have arbitrarily chosen $\widehat{N}=4$, i.e., one zero-energy symbol every four information bits (figure 2), in order to keep the simulation time small.

Figure 3 shows the BER performance of the proposed receiver as a function of the ratio between the mean energy per bit at the receiving end $\left(E_{\mathrm{b}}\right)$, i.e., taking into account zeroenergy bit insertion, and noise power spectral density $\left(N_{0}\right)$ if a two element uniform linear array is considered. In this figure, the proposed detector is compared to a single element multiuser detector. Moreover, in this figure and in all the others the BER performance of a single user rake receiver, employing the full array gain, is shown as the lower bound limit. Figure 4 shows the BER performance of the proposed multiuser detector in the case of ideal power control and for a relative power difference of $20 \mathrm{~dB}$ between the desired user signal and the interfering signals. The BER performance obtained for a beamformer rake receiver with the same array structure and the same number of interfering users is also shown in the figures for comparison purposes. The good behavior of the array multiuser detector is evident in figures 3 and 4 . Moreover, figure 4 highlights the fact that the BER performance of the proposed multiuser detector is almost the same even for high values of interference. This result demonstrates a remarkable near-far resistance of the proposed detector, i.e., a high energy level of interfering users does not affect system performance. This result confirms decorrelating detector properties in MAI [9] also for SWA implementations.

In figure 5, the proposed receiver is compared with a two element sliding decorrelator wherein replicas are combined according to the Maximum Ratio algorithm: these results demonstrate that optimum combining allows a slight perfor- 


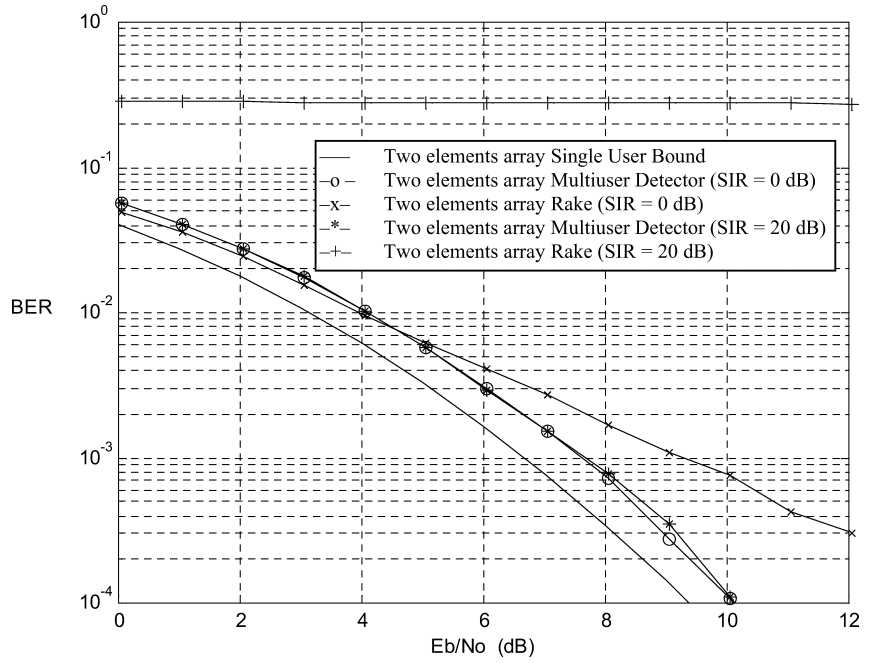

Figure 4. Two elements antenna receiver BER comparisons (multiuser detector and rake performance with power unbalance between the desired user signal and the interfering signals equal to 20 and $0 \mathrm{~dB}$, i.e., ideal power control).

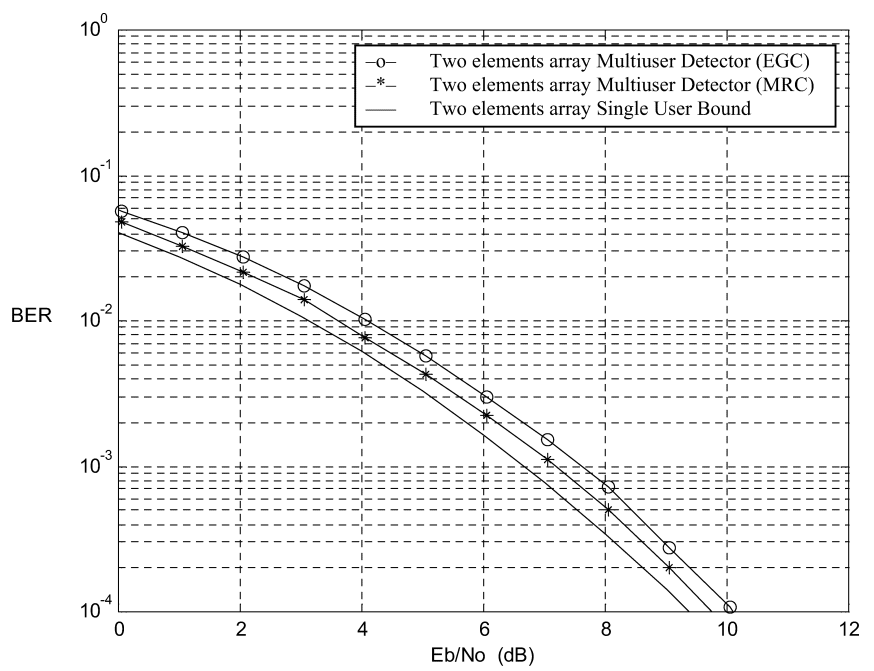

Figure 5. Two elements antenna receiver bit error rate comparisons (ideal power control, multiuser detector performance with MRC and EGC algorithms).

mance improvement, by requiring the knowledge of signal replica amplitudes.

The results shown in figure 6 concern the case of a fourelement array. This figure proves that the BER performance is related to the number of array elements. In this figure, the single user beamformer-rake receiver performance in the case of a four element array is shown as the lower bound limit. For comparison purposes, a single element multiuser detector performance is also shown. The figure also shows the performance gain caused by the increase in the number of array elements: in particular, $3 \mathrm{~dB}$ gain is achieved for each doubling of the number of antenna elements. Since the complexity increase due to higher number of elements is negligible as reported in section 2 , this performance gain is quite interesting.

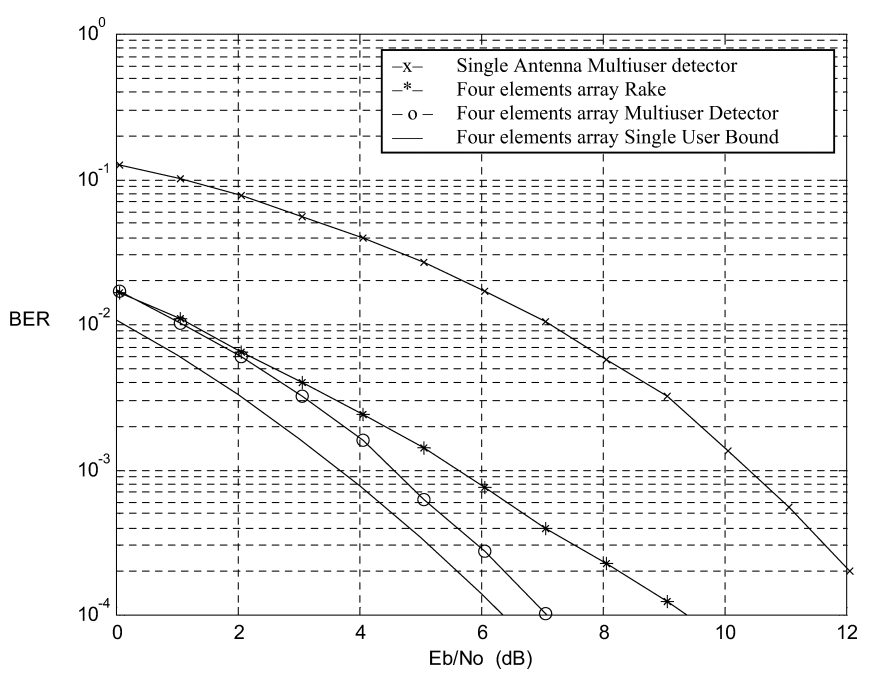

Figure 6. Two and four elements antenna receiver bit error rate comparisons (ideal power control, multiuser detector and rake performance).

\section{Concluding remarks}

In this paper we presented a low complexity multiuser receiver employing a linear beamformer followed by a Sliding Window Decorrelator for DS/CDMA up-link communications. The proposed architecture avoids linear filter realization of the decorrelator and operates with relatively short data frames instead of the complete information sequence. The near-far resistance of our receiver and its good behavior with respect to the classical rake receiver have been verified by means of computer simulations in the case of a slowly frequency-selective Rayleigh fading channel.

By using time and space processing and decorrelating techniques, it has been demonstrated that the proposed receiver has a fairly good near-far resistance and achieves excellent performance in a multipath fading environment with a reasonable improvement in comparison with a single-antenna receiver. Finally, if a greater number of antenna elements is considered, the BER performance of the proposed receiver improves significantly while the implementation complexity increases only slightly.

\section{Acknowledgements}

The authors would like to thank Dr. William Sanders for his useful suggestions and helpful cooperation during the review process of the paper.

\section{References}

[1] M. Bueher and B.D. Woerner, Analysis of adaptive multistage interference cancellation for CDMA using an improved Gaussian approximation, IEEE Transactions on Communications 44 (October 1996) 13081321.

[2] E. Del Re, R. Fantacci, S. Morosi and G. Vivaldi, A low-complexity multiuser detector for up-link CDMA QPSK mobile radio communications, Wireless Networks 5(6) (1999) 461-465. 
[3] R.E. Donovan, S.Y. Yoon, S.E. Hong, J. Ahn and H.S. Lee, Pilot symbol aided coherent decorrelating detector for up-link CDMA mobile radio communication, Electronics Letters 30(12) (1994) 929-930.

[4] 3rd Generation Partnership Project (3GPP), Technical Specification Group (TSG), Spreading and Modulation (FDD), TS 25.213 V3.3.0 (June 2000).

[5] 3rd Generation Partnership Project (3GPP), Technical Specification Group (TSG), Physical Layer Procedures (FDD), TS 25.214 V3.3.0 (June 2000).

[6] GSM Recommendation 05.05 (Ver. 3.7.0).

[7] M.J. Juntti, B. Aazhang and J.O. Lilleberg, Iterative implementation of linear multiuser detection for dynamic asynchronous CDMA systems, IEEE Transactions on Communications 46 (April 1998) 503-508

[8] R. Lupas and S. Verdù, Near-far resistance of multiuser detectors in asynchronous channels, IEEE Transactions on Communications 38 (April 1990) 496-508.

[9] S. Miller and S. Schwartz, Integrated spatial temporal detectors for asynchronous Gaussian multiple access channels, IEEE Transactions on Communications 43 (February/March/April 1995) 396-411.

[10] Mobile Station-Base Station Compatibility Standard for Dual-Mode Wideband Spread Spectrum Cellular System IS-95A, Telecommunications Industry Association, TIA/EIA, Washington, DC (1995).

[11] S. Verdú, Multiuser Detection (Cambridge University Press, 1998).

[12] X. Wang and H. Poor, Space-time multi-user detection in multipath CDMA channels, IEEE Transactions on Signal Processing 47 (September 1999) 2356-2374.

[13] S.S.H. Wijayasuria, G.H. Norton and J.P. McGeehan, Sliding window decorrelating algorithm for DS-CDMA receivers, Electronics Letters 28(17) (1992) 1596-1598.

[14] F. Zheng and S. Barton, Near-far resistant detection of CDMA signals via isolation bit insertion, IEEE Transactions on Communications 43 (February/March/April 1995) 1313-1317.

Enrico Del Re received the Dr. Ing. degree in electronics engineering from the University of Pisa, Pisa, Italy, in 1971. Until 1975 he was engaged in public administration and private firms, involved in the analysis and design of the telecommunication and air traffic control equipment and space systems. Since 1975 he has been with the Department of Electronics Engineering of the University of Florence, Florence, Italy, first as a Research Assistant, then as an Associate Professor, and since 1986 as Professor. During the academic year 1987-1988 he was on leave from the University of Florence for a ninemonth period of research at the European Space Research and Technology Centre of the European Space Agency, The Netherlands. His main research interest are digital signal processing, mobile and satellite communications and communication networks, on which he has published more than $150 \mathrm{pa}-$ pers, in international journals and conferences. He is the Co-editor of the book Satellite Integrated Communications Networks (North-Holland, 1988), one of the authors of the book Data Compression and Error Control Techniques with Applications (Academic Press, 1985) and the editor of the books Mobile and Personal Communications (Elsevier, 1995) and Software Radio Technologies and Services (Springer, 2001). He has been the Chairman of the European Project COST 227 "Integrated Space/Terrestrial Mobile Networks" and the EU COST Action 252 "Evolution of satellite personal communications from second to future generation systems". He received the 1988/89 premium from the IEE (UK) for the paper "Multicarrier demodulator for digital satellite communication systems". He is the head of the Digital Signal Processing and Telematics Laboratory of the Department of Electronics and Telecommunications of the University of Florence. He is a member of the Executive Board of the Italian Interuniversity Consortium for Telecommunications (CNIT).

Romano Fantacci received the Dr. Ing. degree in electronics engineering and the Ph.D. degree in telecommunication engineering, both from the University of Florence, Florence, Italy, in 1982 and 1987, respectively. In 1982 he joined the Electgronics and Telecommunications Department, University of Florence, first as Researcher, then as an Associate Professor and currently as Full Professor of Telecommunications Networks. During the first stage of his research activity, he worked on satellite communication systems. In particular, he has been involved in several European Space Agency (ESA) and INTELSAT advanced research projects. He is the author of several pubblications, some of which have been pubblished in the most acknowledged journals in communication science. He has also been involved in several European and nationwide research projects. His present research interests involve digital communications, computer communications, queueing theory, fast packet switching and mobile communication networks.

Simone Morosi received the Dr. Ing. degree in electronics engineering in 1996 and the Ph.D. degree in information and telecommunication engineering in 2000 from the University of Florence, Florence. Since 2000 he has been with the Department of Electronics and Telecommunications of the University of Florence, as a Research Assistant. His present research interests involve Code Division Multiple Access communications, Multiuser Detection Techniques, Adaptive Antenna Array systems, Turbo Coding, Digital Communications and 3rd generation mobile communication standards.

Stefano Marapodi received the Dr. Ing. degree in electronics engineering from the University of Florence, Florence, Italy, in 1997. He is an independent consultant with experience in software design and training; since 1993 works for software and telecommunication company. Since 2000 he has been with University of Florence as member of a research project focused on 3rd generation mobile communication systems, sponsored by Marconi Comms. 